\title{
Dexverapamil to modulate vinblastine resistance in metastatic renal cell carcinoma
}

\begin{abstract}
Multidrug resistance (MDR) in a variety of human tumours such as renal cell carcinoma (RCC) is thought to be caused by expression of the MDRI gene and may be reversed by applying modern chemosensitisers such as dexverapamil, which inhibit the $M D R I$ gene product P-glycoprotein. This preliminary report gives information on a clinical study complying with good clinical practice regulations in patients with advanced RCC. The final evaluation is pending. Vinblastine, if anything the most effective chemotherapeutic agent (5-day continuous regimen), was combined with oral dexverapamil (6 times per day) as a chemosensitiser and dexamethasone to increase dexverapamil tolerance. All patients had histologically proven $\mathrm{RCC}$, which was metastatic and progressive at study entry. The statistical design featured a pre-study regimen of two cycles of vinblastine alone followed by evaluation. If no response was documented, with all patients thus serving as their own control, dexverapamil and dexamethasone were added for three cycles of combination therapy. Having obtained institutional permission from the ethical review committee, we enrolled patients of whom 25 qualified for the combined-treatment arm; 13 patients finished the study, 5 patients failed to complete all treat-
\end{abstract}

Work presented at the Satellite Symposium "Dexverapamil: a clinical approach to circumvention of multidrug resistance" held at the 1 st International Conference on Reversal of Multidrug Resistance in Cancer, St. Gallen, Switzerland, 1-3 September 1994

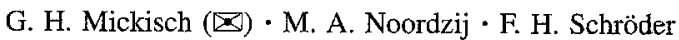

Department of Urology, AZR - Dijkzigt, Erasmus University, Dr. Molewaterplein 40, NL-3015 GD Rotterdam, The Netherlands Fax: $31 / 104635838$

A. v. d. Gaast

Department of Medical Oncology, Erasmus University and Academic Hospital, 3015 GD Rotterdam, The Netherlands

P. Gebreamlack · K. U. Köhrmann

Department of Urology, Mannheim Hospital, University of Heidelberg, Germany

E. Mogler-Drautz $\cdot$ H. Kupper

Dept of Clinical Oncology, Knoll AG, Ludwigshafen, Germany ment cycles ( 1 because of treatment-related toxicity, 3 for personal reasons, not related to treatment, 1 for tumourrelated reasons) and 7 patients were at too early a stage for evaluation. Altogether, $61 \%$ of all patients tolerated a dose of dexverapamil of at least $2400 \mathrm{mg} /$ day with peak serum levels reaching, in some cases, approximately $8 \mu \mathrm{M}$ (the sum of dexverapamil plus nordexverapamil levels). WHO grade 3 and 4 toxicities were mainly myelosuppression $(5 / 18)$. The combination of $1.4 \mathrm{mg} \mathrm{m}^{-2}$ day-1 vinblastine plus dexverapamil was generally felt to be safe and well tolerated. One partial response and 7 stable diseases were noted in this heavily pretreated study population. Fourhourly administration of dexverapamil in combination with dexamethasone plus escalation to the individually tolerated doses have permitted increases in serum levels of dexverapamil.

Key words Dexverapamil $\cdot$ Multidrug resistance $\cdot$ Renal cell carcinoma $\cdot$ Clinical study $\cdot$ Chemosensitisation

\section{Introduction}

Renal cell carcinoma ( $\mathrm{RCC}$ ) is the third most common urological malignancy and accounts for approximately $3 \%$ of all adult tumours. The treatment of choice for nondisseminated disease relies on surgery. The 5-year survival of all stages reached $40 \% 40$ years ago, 50\% 20 years ago, and amounted to $60 \%$ in the recent literature. It is widely accepted that, in addition to refinement of operative strategies and general hospital care, the use of ultrasound in medicine has been of paramount importance, leading to earlier detection of tumours that are more frequently amenable to surgical resection. However, further improvement in the prognosis of RCC is most likely to depend on the eventual development of a systemic treatment for the still high number of patients presenting with metastatic disease.

Therapeutic options in advanced stages, including hormonal therapy or immuno- or chemotherapy, have no 
proven efficacy, and there is an abundance of recent investigations using innovative forms of immunotherapy, gene therapy, and chemosensitisation-enhanced chemotherapy. The latter strategy is the focus of this paper.

Ever since the discovery that certain toxic drugs could be used to treat cancer, clinical researchers have been faced with the problem of drug resistance. It prevents response in some tumours such as RCC and develops at an alarmingly high frequency in some neoplasms that initially appear to be suitable for chemotherapy.

Oncologists often ask why the cancers they treat with chemotherapy readily display or develop resistance to powerful anticancer drugs when their toxicity to normal tissues such as bone marrow and the gastrointestinal tract persists. In the search for answers to perplexing questions such as this, a cellular protein termed P-glycoprotein (Pgp) and encoded by the MDR1 gene (reviewed in Gottesmann and Pastan 1993) has been discovered. Study of drugresistant cells, derived from patients and propagated in tissue culture, gave the surprising result that all of these different chemotherapeutic agents, which are easily soluble in cell membranes and enter cells by simple diffusion, were failing to accumulate in such resistant cancer cells. Furthermore, the low cellular drug levels resulted from the presence of an energy-dependent pump at the cell surface, which rapidly removed the drugs as soon as they started to become internalised. A molecular sump pump rescued these cancer cells from natural-product cytotoxic agents.

For nearly half a century since the inception of an organised drug-screening programme to improve cancer chemotherapy, efforts have been largely compound-oriented (sensitive drug screens) then disease-oriented (tumour panel), metastasis-oriented (orthotopic animal models) and recently MDR-oriented (relevant resistant drug screens). In fact, knowledge of the Pgp-driven mechanism of drug resistance has resulted in a bold initiative in chemosensitisation-enhanced chemotherapy to intensify classical chemotherapy by virtue of inhibiting the underlying resistance factor, e.g. Pgp (reviewed in Gottesman and Pastan 1993).

Based on an encouraging preclinical analysis using RCC lines (Fojo et al. 1987), primary RCC cultures (Mickisch et al. 1990) or transgenic mice expressing the MDRI gene at levels present in RCC (Mickisch et al. 1991), a clinical study was initiated in patients with RCC. This is a tumour entity for which there are at present no reliable therapeutic options in advanced stages and which almost always expresses Pgp (Fojo et al. 1987; Kakehi et al. 1988). Vinblastine, if anything the most effective chemotherapeutic agent, is combined with dexverapamil, a prototype second-generation chemosensitiser, to test for chemomodulation. Since comprehensive animal experimentation indicated that dexamethasone markedly increased dexverapamil tolerance (Gottesman et al. 1994), this drug is also included in the protocol. The main study objectives for evaluation were toxicity and efficacy of dexverapamil in conjunction with vinblastine and dexamethasone in patients with vinblastine-resistant RCC. Additional parameters comprised an MDR1 expression analysis of the primary tumour and serum pharmacokinetics of dexverapamil, vin-
Table 1 Dexverapamil in metastatic renal cell carcinoma (RCC). Patient and tumour characteristics

\begin{tabular}{lc}
\hline Characteristic & \\
\hline Total no. patients & 18 \\
Age (years) & \\
Median & 61 \\
$\quad$ Range & $41-68$ \\
Sex & \\
Female & 4 \\
Male & 14 \\
Prior therapies of RCC (not exclusive) & \\
Nephrectomy & 14 \\
Chemotherapy & 0 \\
$\quad$ Immunotherapy & 2 \\
$\quad$ None & 4 \\
Site of metastasis (not exclusive) & \\
Lung & 15 \\
Liver & 2 \\
Lymph node & 6 \\
Bone & 4 \\
Other & 3 \\
\hline
\end{tabular}

blastine and the combination of the two drugs. Furthermore, an in vitro assay to determine the ability of patient serum containing dexverapamil to circumvent MDR of RCC lines was carried out at different assay times during the study. These results will be reported separately.

\section{Patients and methods}

Patients with a clinical and histological diagnosis of advanced RCC were studied in a two-stage (sequential) phase-II investigation complying with good clinical practice regulations.

\section{Eligibility requirements}

The clinical work-up was always based on complete blood count, chemistry, ECG, assessment of the left ventricular ejection fraction, chest $\mathrm{X}$-rays and computed tomography (CT) of the abdomen, which also served for the evaluation of the treatment results. Additional diagnostic procedures such as bone scans and $\mathrm{CT}$ of the thorax, brain etc. were performed if necessary. Histology relied on a surgical specimen from the primary tumour if available. In patients not undergoing radical nephrectomy prior to entering the study, a needle (core) biopsy of the tumour mass served to prove the clinical diagnosis of RCC. All participants came from the regular patient population and were referred to us by family physicians, urologists or medical oncologists for further counselling and therapy of metastatic disease. No attempt was made to select for specific characteristics; only patients with a WHO performance score of 3 or more for metastasis to the central nervous system (CNS) were excluded from enrolment in this study. The details are compiled in Table 1.

Informed consent was obtained from all patients before participation in the study, and protocols and consent forms were approved for medical and ethical indications by the institutional protocol review board (MEC 124, 106-1993/12). All patients were recruited between May 1993 and May 1994, and all of them were treated on a general urology ward.

Previous treatments such as surgery or immuno- or chemotherapy were permitted if a minimal waiting period of 8 weeks was observed before study entry and all patients exhibited at least one bidimension- 
Table 2 Dexverapamil in metastatic RCC. Tolerated dexverapamil daily dose ( $n=18$ patients)

\begin{tabular}{ll}
\hline Daily dose $(\mathrm{mg})$ & $\begin{array}{l}\text { Number of patients } \\
\text { (cumulative) }\end{array}$ \\
\hline 1500 & $18(100 \%)$ \\
1800 & $15(83 \%)$ \\
2100 & $15(83 \%)$ \\
2400 & $11(61 \%)$ \\
2700 & $8(44 \%)$ \\
3000 & $6(33 \%)$ \\
\hline
\end{tabular}

Table 3 Dexverapamil in metastatic RCC. Maximal severity in haematological/general toxicities (WHO grading) ( $n=18$ patients)

\begin{tabular}{|c|c|c|c|c|c|c|c|c|c|c|}
\hline \multirow{3}{*}{ Toxicity } & \multicolumn{10}{|c|}{$\begin{array}{l}\text { Number of patients experiencing toxicity } \\
\text { (WHO grades } 0-4 \text { ) }\end{array}$} \\
\hline & \multicolumn{5}{|c|}{ VBL alone } & \multicolumn{5}{|c|}{$\mathrm{VBL}+$ dexverapamil } \\
\hline & 0 & 1 & 2 & 3 & 4 & 0 & 1 & 2 & 3 & 4 \\
\hline White blood cells & 6 & - & 5 & 4 & 3 & 6 & 3 & 1 & 3 & 5 \\
\hline Platelets & 17 & - & 1 & - & - & 15 & 1 & 1 & - & 1 \\
\hline Haemoglobin & - & 1 & 4 & 10 & 3 & - & 1 & 1 & 11 & 5 \\
\hline Nausea/vomiting & 13 & 4 & 1 & - & - & 12 & 5 & 1 & - & - \\
\hline
\end{tabular}

Table 4 Dexverapamil in metastatic RCC. Cumulative cardiovascular effects ( $n=18$ patients)

\begin{tabular}{lcc}
\hline Parameter & VBL alone & VBL + dexverapamil \\
\hline Mean heart rate (beats/min) & $79.9 \pm 6.1$ & $70.7 \pm 6.1$ \\
Mean blood pressure & & \\
$\quad$ Systolic (mm Hg) & $144.5 \pm 17.9$ & $118.8 \pm 13.4$ \\
$\quad$ Diastolic (mm Hg) & $84.9 \pm 10.4$ & $72.0 \pm 8.4$ \\
Mean PQ time (ms) & $157.3 \pm 28.5$ & $168.9 \pm 26.3$ \\
\hline
\end{tabular}

ally measurable metastatic lesion other than bone metastases. Local radiotherapy for painful tumour deposits to bone was acceptable if completed 8 weeks prior to the first treatment cycle, and if the reference tumour lesion was excluded from the radiation field. No concomitant medication involving $M D R I$-related drugs or compounds that may affect cardiac function, such as beta blockers, were allowed for the whole study duration. The left ventricular ejection fraction was assessed at baseline, prior to combination therapy and at the end of the study to have a value $50 \%$ that of age-matched population controls, serving as the threshold for study entry/continuation.

\section{Treatment plan}

The treatment schedule was $2 \mathrm{mg} / \mathrm{m}^{2}$ vinblastine (patients $1-10$; optional dose reduction) amended to $1.4 \mathrm{mg} / \mathrm{m}^{2}$ (patients 11 -study end; optional dose intensification) as a 5-day continuous infusion. Dexverapamil was administered orally six times per day starting with $250 \mathrm{mg} /$ dose on days 0 and 1 with a subsequent daily dose escalation of $6 \times 50 \mathrm{mg}$ up to the individual maximum tolerated dose (days 2-6). In addition, $2 \times 20-\mathrm{mg} /$ day dexamethasone was applied as a short-term infusion (days $0-6$ ). The second and third cycles were continued with the highest tolerated dexverapamil dose from cycle one. Vinblastine and dexamethasone solutions were prepared by the hospital pharmacy individually for each patient in accordance with the manufacturer's instructions, and dexverapamil tablets were provided by Knoll AG, Germany. The cycle duration for each protocol was 3 weeks. All

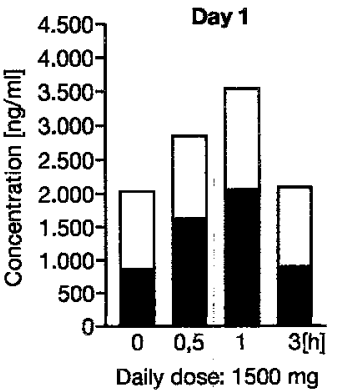

Dexverapamil

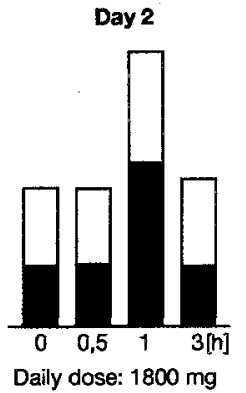

$\square$ Nor-Dexverapami
Day 5

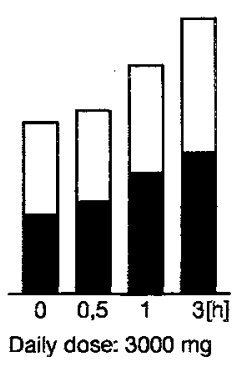

Fig. 1 Dexverapamil and its first metabolite, nor-dexverapamil, serum levels in the patient C. F. undergoing dexverapamil/vinblastine/dexamethasone treatment in accordance with protocol MEC 124, $106-1993 / 12$. Note the combined serum levels surpass $8 \mu \mathrm{M}$ (approximately $4000 \mathrm{ng} / \mathrm{ml}$ )

patients were documented to be metastatic and progressive at study entry.

Electrocardiograms (ECG) of all cycles of combination therapy were obtained every day. If a first-degree atrioventricular block appeared, the dose of dexverapamil was reduced until normalisation of the ECG. Pulse rate and blood pressure were measured every $30 \mathrm{~min}$ after intake of any dexverapamil medication, and routine haematology, blood chemistry and urine analysis were recorded during all treatment cycles.

\section{Statistics}

The statistical design featured a prestudy of two cycles of vinblastine alone followed by tumour evaluation. If no response was documented, dexverapamil and dexamethasone were added for three cycles of combination therapy, all patients thus serving as their own control.

The statistical evaluation followed the two-stage design by Gehan (1961). A sample size of 14 patients thus guaranteed that a true response rate of $20 \%$ would cause at least one objective response ( $\beta$ error $=0.05$ ). In this scheme, the trial would have been discontinued if no response had been observed after 14 patients, with further enrolment depending on the number of patients needed to estimate the real response rate with a required precision of $10 \%$ in terms of the standard error (maximum number $=25$ ).

\section{Results}

We report here preliminarly on 18 patients who were entered into the main study protocol (combined-treatment arm). Thirteen of them are fully evaluable; 5 patients failed to complete all treatment cycles but could be assessed for side-effects only. In this particular trial the investigators opted for the administration of dexverapamil six times per day and used dexamethasone to increase dexverapamil tolerance further. Table 2 summarises the accumulated dexverapamil intake for all 18 evaluable patients. It is obvious that our administration regimen allows for a significant increase in daily dosage.

We then examined how this progress in drug availability would translate into serum levels of dexverapamil. Figure 1 provides an example of this part of the pharmacological investigations, clearly showing the increase in peak serum levels of dexverapamil and its metabolite nor-dexverapa- 
Table 5 Dexverapamil in metastatic RCC. Individual cardiovascular side-effects ( $n=18$ patients) (VBL vinblastine, $A V$ atrioventricular)

\begin{tabular}{lrl}
\hline Parameter & VBL alone & VBL + dexverapamil \\
\hline Heart rate & 16 & 7 \\
$>60$ beats $/ \mathrm{min}$ & 2 & 6 \\
$50-59$ beats/min & 0 & 4 \\
$40-49$ beats $/ \mathrm{min}$ & 0 & 1 \\
$<40$ beats $/ \mathrm{min}$ & & \\
Blood pressure systolic & 18 & 5 \\
$>100 \mathrm{~mm} \mathrm{Hg}$ & 0 & 8 \\
$90-99 \mathrm{~mm} \mathrm{Hg}$ & 0 & 4 \\
$80-89 \mathrm{~mm} \mathrm{Hg}$ & 0 & 1 \\
$<80 \mathrm{~mm} \mathrm{Hg}$ & 0 & 5 \\
AV block first degree & 0 & 0 \\
AV block higher degree & 0 & 0 \\
AV rhythm & 0 &
\end{tabular}

Table 6 Dexverapamil in metastatic RCC. Treatment efficacy $(n=13$ patients) ( $C R$ complete response, $P R$ partial response, $S D$ stable disease, $P D$ progressive disease

\begin{tabular}{lcc}
\hline Response & VBL alone & VBL + dexverapamil \\
\hline CR & 1 a & - \\
PR & - & 1 \\
SD & 9 & 7 \\
PD & 3 & 5 \\
Total & 13 & 13 \\
\hline
\end{tabular}

a Later became progressive and entered the main study to reach stable disease

mil, which has the same modulating activity as dexverapamil (Merry et al. 1989; Häußermann et al. 1990). It is noteworthy that the sum of the serum levels of these drugs surpasses $8 \mu \mathrm{M}$, a blood concentration previously thought to be difficult to reach. The true extent of this pharmacological advantage can be gleaned from earlier measurements using racemic verapamil that never exceeded $1.5 \mu \mathrm{M}$ (Ozols et al. 1987), and all of the patients remained in intensive-care units.

Moreover, the question arose whether the increased serum levels of dexverapamil would reveal unacceptable side-effects, thus impeding clinical applicability of this approach. In Tables 3-5, we compare haematological and general toxicities, general cardiovascular effects and individual cardiovascular side-effects of vinblastine monotherapy (pre-study) and of the combined medication (main study). There is no doubt that we encountered some adverse effects, mainly myelosuppression, with our regimen that were, in principle, strictly associated with the chemotherapeutic agent vinblastine. This led to a protocol amendment of the vinblastine starting dose to $1.4 \mathrm{mg} / \mathrm{ml}$ instead of the original $2 \mathrm{mg} / \mathrm{m}^{2}$. However, this toxicity was manageable, with some participants $(n=6)$ requiring hospitalisation to cope with the side-effects and subsequent dose reductions and/or delay in receiving further treatment cycles.

Putative dexverapamil-related drawbacks were predominantly cardiovascular, mostly mild in nature and definitely not life-threatening, and led to dose reductions in 5 patients experiencing an atrioventricular block grade I. In general, $1.4 \mathrm{mg} \mathrm{m}^{-2}$ day $^{-1}$ vinblastine plus dexverapamil was felt to be safe and reasonably well accepted. There were 5 patients who failed to complete all treatment cycles. In 1 case, myelotoxicity with severe septic complications precluded further participation in the study. In 3 patients treatment was discontinued because of progressive disease or deterioration of the performance status, and 1 patient with advanced RCC died of cardiopulmonary insufficiency due to dislocation of a caval tumour thrombus.

Having established that very high serum levels of dexverapamil did not produce excessive toxicity in a general patient population suffering from metastatic RCC, we also wished to assess efficacy of this particular regimen. Table 6 shows 1 partial response and 7 patients with stable disease in this pretreated study cohort that was documented to be progressive at study entry and refractory to vinblastine monotherapy. The partial response occurred in a thoracic wall metastasis including recalcification of the rib lesion. There was a tendency of lung metastases to decrease in 3 patients staying within the broad limit of stable disease. Hence, it appears that the chosen regimen and dosages result in marginal activity in $\mathrm{RCC}$ with this innovative treatment concept.

\section{Discussion}

MDR, mediated by Pgp and encoded by the $M D R I$ gene, is one of the best understood mechanisms of resistance to cytotoxic drugs. A growing body of evidence indicates that expression of $M D R I$ contributes to clinical resistance to chemotherapy. Pgp in normal tissues may be important in the excretion of MDR-related anticancer agents as well as many other compounds.

Several clinical trials (overviewed in Sikic 1993; Raderer and Scheithauer 1993; Fan et al. 1994; Mickisch 1994; Lehnert 1994) have attempted to modulate MDR by coadministration of non-cytotoxic inhibitors of Pgp, such as verapamil and cyclosporin A. Interpretation of these studies is complicated by a number of factors: (a) adequate concentrations of many potential modulation agents may not be achievable because of inherent toxic side-effects (e.g. hypotension and heart block with verapamil), (b) the pharmacokinetic consequences of the drug interactions that are produced have not been well characterised (drug levels of both modulator and cytotoxins), (c) the modulating drug may not be bioavailable in vivo because of binding to factors such as serum proteins (e.g., amiodarone), (d) proper controls may not be included (proven clinical resistance to prior therapy or a randomised control group without the chemosensitiser), (e) measurement of MDR1 expression by tumour cells may not be available, and (f) even if the tumour expresses MDR1, redundant mechanisms of resistance may be present in tumour cells.

Nonetheless, the prognostic significance of $M D R I$ expression and anecdotal observations of clinical remissions 
with modulation of MDR have led to sustained interest in this area. At present, attention is being focused on clinical investigations using second-generation chemosensitisers, such as dexverapamil or others that have been selected for high efficacy or low toxicity in circumventing MDR.

Clinically, there is profound scepticism about the use of classical chemotherapy in RCC, but since there are no reliable therapies for metastatic disease, innovative approaches including chemosensitisation seem warranted. Recent and ongoing clinical studies in RCC have been conducted under phase I/II conditions. Hence, the need for well-controlled or randomised trials to evaluate these chemomodulators has become imperative. So far, three investigations in RCC have been reported, but unfortunately, the initial results have not been very rewarding. There was one attempt to circumvent MDR in 15 patients with $\mathrm{RCC}$ by combining bolus vinblastine with infusional cyclosporin A (Rodenburg et al. 1991). Median cyclosporin plasma levels reached a modest $5668 \mathrm{ng} / \mathrm{ml}$. Thus, toxicity was described as minimal, and so was efficacy.

Lately, dexniguldipine, a dihydropyridine derivative, was administered orally in conjunction with i.v. doxorubicin, a cytotoxic agent without activity in RCC when given as monotherapy. Thirty patients were recruited for this study (Gehling et al. 1993). Gastrointestinal toxicity was considerable, preventing a reasonable dose escalation.

More recently, in a pilot study, continuous i.v. vinblastine and oral dexverapamil, the $R(+)$ stereoisomer of racemic verapamil, were administered concomitantly to 12 patients (Overmoyer et al. 1993) with continuation of patient accrual. Response rates have not yet been reported, but the study design featuring four equal daily doses of dexverapamil led to plasma levels associated with cardiovascular side-effects so that most patients did not tolerate more than $1500 \mathrm{mg}$ dexverapamil/day.

On the basis of a preclinical analysis in which dexverapamil was added during the long-term exposure of RCC lines to vinblastine (Mickisch et al. 1990) it was decided to apply vinblastine in a continuous infusional regimen in conjunction with oral dexverapamil. In addition, the dexverapamil medication was distributed over six equal daily doses and dexverapamil tolerance was further enhanced by dexamethasone support. Hitherto, reports have mainly described dexverapamil medication broken into four equal daily doses without dexamethasone support (Bissett et al. 1991; Schumacher et al. 1991; Overmoyer et al. 1993), and the maximum tolerated dose for dexverapamil ranged from $1200 \mathrm{mg} /$ day to $1500 \mathrm{mg} /$ day. There have been two preliminary reports on four-hourly dexverapamil medication, either in conjunction with etoposide, prednison, oncovin (vincristine), cyclophosphamide, hydroxydoxorubicin (EPOCH) chemotherapy (Wilson et al. 1993) or with taxol (Tolcher et al. 1994). Both observations document feasibility and reasonable tolerance for this mode of application. Although there is scarce experimental evidence suggesting that some steroid hormones may be pumped by Pgp (Ueda et al. 1992; Van Kalken et al. 1993), any significant contribution of dexamethasone to reverse MDR under clinical circumstances remains highly improbable.
When evaluating this regimen, important progress was made in dexverapamil tolerance (Table 2) and blood serum levels (Fig. 1) and some activity in RCC (Table 6) was achieved.

The dose and schedule of chemotherapy indicate factors that might strongly affect the therapeutic value of chemosensitisers. Continuous infusion of various MDR drugs alone has been shown to reinduce remissions in a number of patients with drug-refractory lymphomas in two independent studies, and some experimental studies proved long-term drug exposure to be superior in subduing drug resistance in Pgp-positive cancer cell lines (overviewed in Sikic 1993). Notably, all clinical studies where chemosensitisers have helped to amend chemotherapeutic efficacy to date have employed continuous infusion of cytotoxic agents (overviewed in Mickisch 1994). Obviously, a certain amount of cytotoxic drug is needed at the site of molecular action to kill cancer cells, and it seems conceivable that blocking of the Pgp function may contribute to such intracellular threshold concentrations being achieved more readily if chemosensitisers are added to high doses of MDR agents.

To assess the activity of chemosensitisers unequivocally, patients need to be treated with the identical cytotoxic regimen, with or without the chemosensitiser. Two strategies appear feasible: one is to perform an interpatient comparison in prospective, randomised trials. This classic phase III design is mainly suitable if a standard therapy were to be evaluated against a new approach that had been active in previous phase-II investigations. Alternatively, intrapatient comparison, i.e. treatment of patients with chemotherapy alone followed by the identical chemotherapy supplemented with chemosensitisation, once drug refractoriness has been established, can be used. The latter approach, which was also implemented in our phase II study, appears to offer some advantages over randomised comparisons: (a) clinical resistance to a particular chemotherapy has been documented; (b) small patient numbers are required to determine whether a chemosensitiser is capable of overcoming drug resistance, and (c) only patients who are known to be unresponsive to a particular (standard) treatment risk experiencing toxicities from an experimental chemosensitiser. However, the broad application of this step depends on the demonstration of substantial effectiveness in phase I-II trials.

There is no doubt that trials delineating the activity of a chemosensitiser should be complemented by various collateral studies to enhance the amount of information gained from such studies and to facilitate the proper interpretation of results. Issues that appear to be indispensable in this respect comprise (a) $M D R I$ expression analysis in tumour specimens, even though it is not known at present what level of MDRl expression is needed to confer clinical drug resistance and, thus, this cannot reasonably be used for discrimination or as an exclusion criterion, (b) measurement of blood serum levels of the chemosensitiser, (c) testing of patient serum containing the chemosensitiser for the ability to reverse MDR in appropriate in vitro models, and (d) pharmacokinetics of chemotherapeutic 
agents with and without the chemosensitiser to discern whether an observed clinical activity can be traced back to effective blocking of Pgp in cancer cells rather than merely being due to pharmacological interaction, leading to elevated concentrations or prolonged retention of the cytotoxic agents. Our comprehensive protocol in RCC has addressed all of these points in a single study.

There is now preliminary evidence to suggest that the concept of MDR reversal can function not only in experimental sytems, but also in cancer patients. This area of clinical research is still in its infancy, and the lack of more impressive data seems little reason to discourage further development. Novel strategies will appear on the horizon that may be able to improve the clinical utility of MDR reversal. However, effective clinical use of chemosensitisers is a complex and difficult task, and much work must still be done to optimise this application further. For the time being, clinical MDR reversal continues to be an experimental approach and should not be pursued outside the context of controlled trials, strictly adhering to the standards of clinical drug development.

\section{References}

Bissett D, Kerr DJ, Cassidy J (1991) Phase I and pharmacokinetic study of D-verapamil and doxorubicin. Br J Cancer 64:1168-1171

Fan D, Beltran PJ, O'Brian CA (1994) Reversal of multidrug resistance. In: Kellen JA (ed) Reversal of multidrug resistance in cancer. CRC Press, Boca Raton, Fl, pp 93-125

Fojo AT, Shen D-W, Pastan I, Gottesman MM (1987) Intrinsic drug resistance in kidney cancers is associated with expression of a human multidrug resistance gene. I Clin Oncol 5:1922-1927

Gehan EA (1961) The determination of the number of patients required in a preliminary and a follow-up trial of a new chemotherapeutic agent. J Chronic Dis 13:346-353

Gehling U, Weimar C, Schuler U, Rathgeb R, Ehninger G, Schumacher K, Havemann K (1993) A pilot study with dexniguldipine and doxorubicin in patients with metastatic hypernephroma. Onkologie 16 [Suppl]:25

Gottesman MM, Pastan I (1993) Biochemistry of multidrug resistance mediated by the multidrug transporter. Annu Rev Biochem 62: $385-427$

Gottesman MM, Mickisch GH, Pastan I (1994) In vivo models of P-glycoprotein-mediated multidrug resistance, In: Goldstein L, Ozols (eds) Anticancer drug resistance. Kluwer, Norwell, Massachusetts, p 103

Häußermann K, Benz B, Gekeler V, Schumacher K, Eichelbaum M (1990) Effects of verapamil enantiomers and major metabolites on the cytotoxicity of vincristine and daunomycin in human lymphoma cell lines. Eur J Clin Pharmacol 40.53-59
Kakehi Y, Kanamaru H, Yoshida O, Ohkubo H, Nakanishi S, Gottesman MM, Pastan I (1988) Measurement of multidrug resistance messenger RNA in urogenital cancers; elevated expression in renal cell carcinoma is associated with intrinsic drug resistance. J Urol 139:862-865

Lehnert M (1994) Reversal of P-glycoprotein-associated multidrug resistance: from bench to bedside. Onkologie 17:8-15

Merry S, Flanigan P, Schlick E, Freshney RI, Kaye SB (1989) Inherent adriamycin resistance in a murine tumour line: circumvention with verapamil and norverapamil. Br J Cancer 59:895-897

Mickisch GH (1994) Chemoresistance of renal cell carcinoma: 1986-1994. World J Urol 12:214-223

Mickisch G, Kössig J, Keilhauer G, Schlick E, Tschada R, Alken P (1990) Effects of calcium antagonists in multidrug resistant primary human renal cell carcinomas. Cancer Res 50:3670-3674

Mickisch G, Merlino GT, Galski H, Gottesman MM, Pastan I (1991) Transgenic mice that express the human multidrug resistance gene in bone marrow enable a rapid identification of agents that reverse drug resistance. Proc Natl Acad Sci USA 88:547-551

Overmoyer B, Fox K, Tomaszewski J, Malkowicz S, MacDermott M, Kay A, Spigelman M, Schuchter L (1993) A phase II trial of $R$-verapamil and infusional vinblastine (velban) in advanced renal cell carcinoma (RCC) (abstract). Proc Am Soc Clin Oncol 12:251

Ozols RF, Cunnion RE, Klecker RW Jr, Hamilton TC, Ostchega Y, Parillo JE, Young RC (1987) Verapamil and Adriamycin in the treatment of drug-resistant ovarian cancer patients. J Clin Oncol 5:641-647

Raderer M, Scheithauer W (1993) Clinical trials of agents that reverse multidrug resistance. Cancer 72:3553-3563

Rodenburg CJ, Nooter K, Herweijer H, Seynaeve C, Oosterom R, Stoter G, Verweij J (1991) Phase II study of combining vinblastine and cyclosporin $A$ to circumvent multidrug resistance in renal cell cancer. Ann Oncol 2:305-306

Schumacher K, Ladda E, Bühl K, Weimer A, Eser C, Hamprecht K, Traugott U, Roller E, Schalk KP, Eichelbaum M (1991) $R$-Verapamil, a modulator of multidrug resistance: phase I clinical trial. J Cancer Res Clin Oncol 117 [Suppl]:113

Sikic B (1993) Modulation of multidrug resistance: at the threshold. I Clin Oncol 11:1629-1635

Tolcher AW, Cowam KH, Solomon D, Berg S, Venzon D, Goldspiel B, Barnes $C$, Noone $M$, Gossard $M$, Chang $R$, Ognibeno $F$, O'Shaugnessy JA (1994) A phase I study of paclitaxel (T) with $R$-verapamil (RV) in metastatic breast cancer (MBC). Proc Am Soc Clin Oncol, 13: 349

Ueda K, Okamura N, Hirai M, Tanigawara Y, Saeki T, Kioka N, Komano T, Hori R (1992) Human P-glycoprotein transports cortisol, aldosterone, and dexamethasone, but not progesterone. J Biol Chem 267:24248-24252

Van Kalken CK, Broxterman HJ, Pinedo HM, Feller N, Dekker H, Lankelma J, Giaccone G (1991) Cortisol is transported by the multidrug resistance gene product $\mathrm{P}$-glycoprotein. $\mathrm{Br} \mathfrak{I}$ Cancer 67:284-289

Wilson WH, Bates S, Kang YK, Fojo A, Bryant G, Wittes R, Stevenson MA, Steinberg S, Chabner BA (1993) Reversal of multidrug resistance (mdr-1) with $R$-verapamil and analysis of mdr-1 expression in patients with lymphoma refractory to EPOCH chemotherapy. Proc Am Assoc Cancer Res 3: 212 